\title{
SATELLITE IMAGE ARTIFACTS DETECTION BASED ON COMPLEXITY DISTORTION THEORY
}

\author{
Avid Roman-Gonzalez, Mihai Datcu \\ German Aerospace Center (DLR), Oberpfaffenhofen, 82234 Wessling, Germany \\ avid.roman-gonzalez@dlr.de,mihai.datcu@dlr.de
}

\begin{abstract}
The artifacts detection is a step of data cleaning process. The classical approach is to predict or determine the existence of defects, to model it, and then design a method to detect and correct them. This classical approach is for specific artifacts. The approach presented in this work is using complexity distortion theory to implement a more generic method, thus, this work will aim at developing parameter free methods able to automatically detect artifacts in EO images. We use the Kolmogorov Structure Function as approximation to the Rate-distortion curve and examine how the artifacts can have the same structure.
\end{abstract}

Index Terms - Artifacts detection, Kolmogorov structure function, Kolmogorov complexity, Rate-distortion, lossy compression

\section{INTRODUCTION}

The Earth Observation (EO) images have many applications; they can help in managing effects of natural disasters, to identify mining areas, biodiversity monitoring, rural and urban environment identification, etc. But sometimes the satellite images can be affected by unexpected distortions, artificial structures called artifacts, produced by the instrument itself or due to the different pre-processing steps. These artifacts may hinder the analysis process and could induce to errors in the indexing process, so that the efficiency of these methods decreases. Thus it is necessary to develop methods for artifacts detection. Several methods have been proposed for specific artifacts detection as banding or ringing. However, to cope with artifacts whose model or nature is unknown, we propose a parameter free artifact detection method regardless the model to which they belong. In [1] and [2] the authors show the first results of the implementation of parameter free methods for artifacts detection, one method is to use the rate-distortion analysis and the other method is based on the normalized compression distance, an example of artifacts detection in a satellite image is presented in figure 1, in these works the authors have achieved better results with rate-distortion analysis, so in this paper we propose to use a rate-distortion analysis based on Kolmogorov complexity, which could be called Complexity-Distortion analysis as presented in [6]. The Complexity-Distortion Theory (CDT) is a mathematical framework providing a unifying perspective on media representation [6], also on the Kolmogorov structure function, studied in [3], define that the individual data as expressed by Kolmogorov's structure function [3].

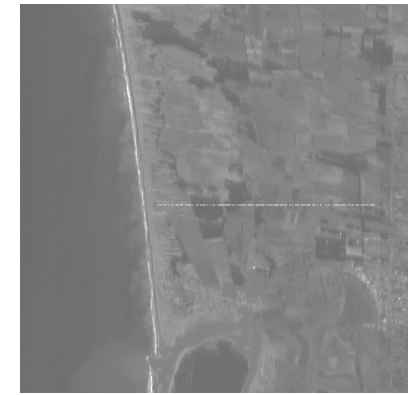

(a) Image with dropout

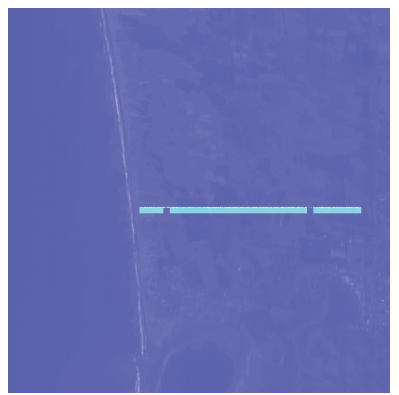

(b) Dropout detection
Fig. 1 Example of artifacts detection

The rest of this paper is constructed as follows: Section 2 presents the theoretical background for the proposed method. Section 3 shows the experiment and results of our work. Finally, Section 4 concludes.

\section{THEORETICAL FRAMEWORK}

In this section we present the theoretical framework on which the proposed method is based, these topics are: Ratedistortion function, Kolmogorov complexity, Kolmogorov's structure function. From these we develop a methodological framework to identify the behavior of structural elements in EO images through curves either rate-distortion function or Kolmogorov structure function.

\subsection{Rate-Distortion Function}

Classical rate-distortion theory was initiated by Shannon. The rate-distortion (RD) function is given by the minimum value of mutual information between source and receiver under some distortion restrictions. 


$$
R(D)=\min _{Q \in Q_{D}} I(p, Q)
$$

Where:

$I(p, Q)$ is the Mutual Information between $\mathrm{p}$ and $\mathrm{Q}$

The RD function shows how much compression (lossy compression) can be used without loss of distortion preset value.

\subsection{Kolmogorov Complexity}

The Kolmogorov complexity $\mathrm{K}(\mathrm{x})$ of a data $\mathrm{x}$ is the length of a shortest program to compute $\mathrm{x}$ on a universal Turing machine, intuitively the Kolmogorov complexity represents the minimal amount information required to generate $\mathrm{x}$ by any effective process [3].

The conditional Kolmogorov complexity K ( $\mathrm{x} \mid \mathrm{y})$ of $\mathrm{x}$ relative to $\mathrm{y}$ is defined as the length of a shortest program to compute $\mathrm{x}$, if $\mathrm{y}$ is furnished as an auxiliary input to the computation

The Kolmogorov complexity has concentrated, however, on data compression, i.e., lossless representation. In [6] they extend it to include distortion for lossy representation and we are going to try to use this lossy representation for calculates an algorithmic rate-distortion function.

\subsection{Kolmogorov's Structure Function}

An approximation of the RD curve using the Kolmogorov complexity theory could be the Kolmogorov Structure Function (KSF). In [3] the authors present an analysis about the Kolmogorov's structure function.

The KFS is an apparent estimator for the maximum resemblance, it is possible to evaluate the smallest set containing $x$, the relation between the data and its representation could be expressed by Kolmogorov's structure function. The original Kolmogorov structure function for a data $\mathrm{x}$ is defined by:

$$
h_{x}(\alpha)=\min _{S}\{\log |S|: S \text { э } x, K(S) \leq \alpha\}
$$

Where: $\mathrm{S}$ is a contemplated model for $\mathrm{x}$.

$\alpha$ is a non-negative integer value bounding the complexity of the contemplated S.

The Kolmogorov's structure function $h_{x}(\alpha)$ tell us all stochastic properties of data $x$. [3].

The Kolmogorov structure function theory also presents the "Best Fit" function (minimal randomness deficiency function).

$$
\beta_{x}(\alpha)=\min _{S}\{\delta(x \mid S): S \text { э } x, K(S) \leq \alpha\}
$$

Where: $\delta(x \mid S)$ is the randomness deficiency of $x$ in $S: \delta(x \mid S)=\log |S|-K(x \mid S)$
The MDL (minimum description length) estimator is defined by:

$$
\lambda_{x}(\alpha)=\min _{S}\{\Lambda(S): S \text { э } x, K(S) \leq \alpha\}
$$

Where: $\Lambda(S)=\log |S|+K(s)$ is the total length of two code of $x$ with help model $S$.

The Kolmogorov structure function is a noncomputable since the Kolmogorov complexity is also a noncomputable function; that is the reason why we use the compression factor as an approximation to complexity. The idea is to use de Kolmogorov structure function as an approximation to the rate-distortion analysis for describe the artifacts behavior and to develop a parameter free artifacts detection method.

In this paper we make an assessment of ratedistortion, complexity-distortion, structure function and its performance as method for artifacts detection, specifically aliasing, banding, dropout and strips.

\section{EXPERIMENTS AND RESULTS}

In this section we are going to present some experiment and results in order to evaluate the behavior of the Kolmogorov Structure Function (KSF) for the different textures, to evaluate if it is possible to discriminate them and after that to use KSF for artifacts detection.

\subsection{Kolmogorov Structure Function for Texture Discrimination}

To evaluate the behavior of the KSF for different textures, we use de Brodatz images databases [8]. In figure 2 we show the textures used for this experiments, and in figure 3 we present the results using KSF.

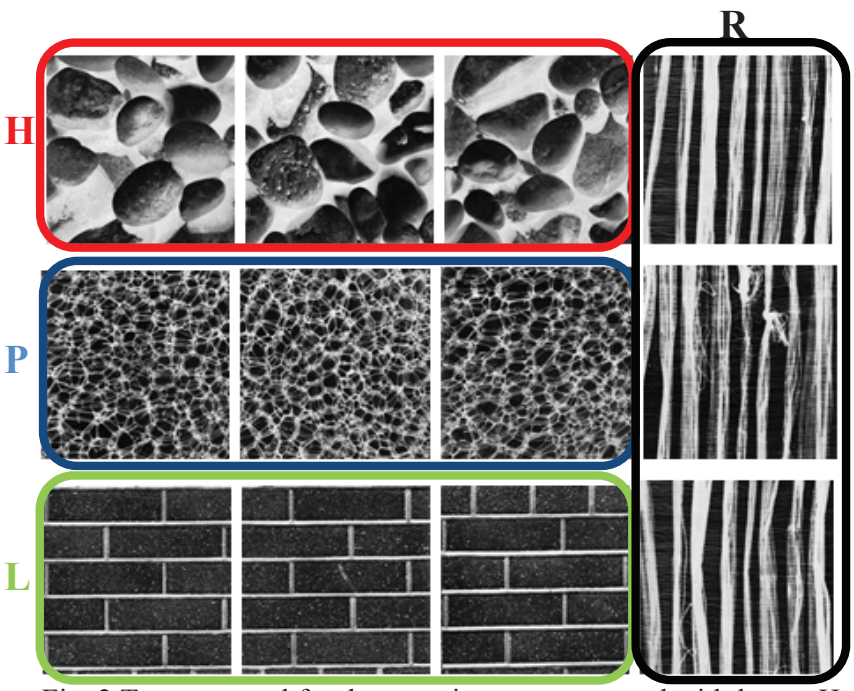

Fig. 2 Textures used for the experiment represented with letters $\mathrm{H}$, $\mathrm{P}, \mathrm{L}$ and $\mathrm{R}$. 


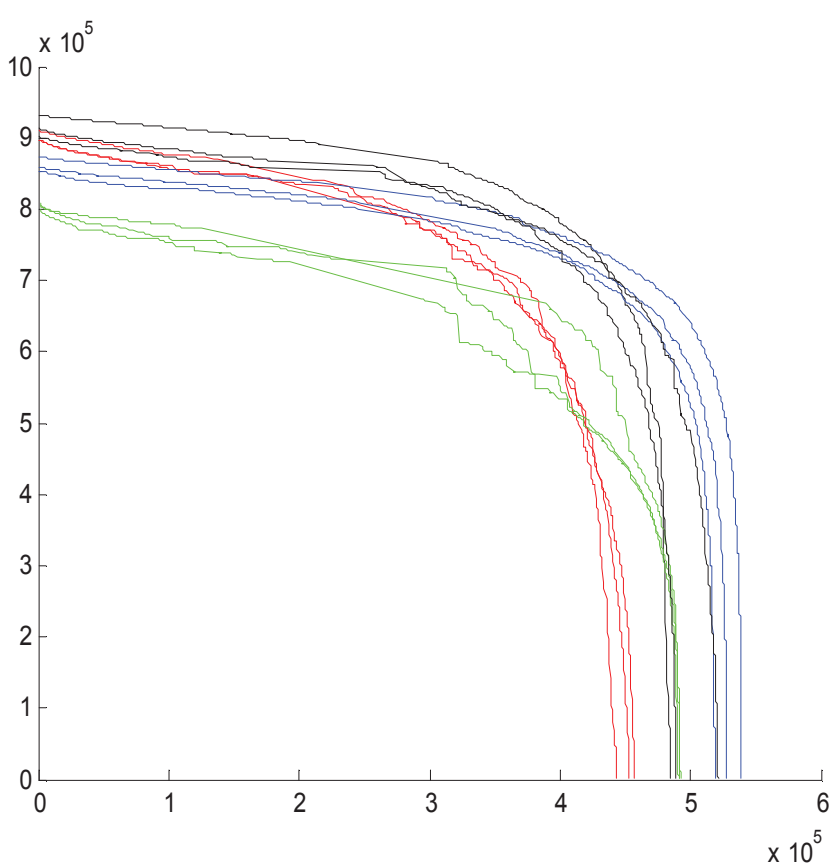

Fig. 3 Kolmogorov Structure Function for the textures presented in figure 2 , the red curve are for the textures $\mathrm{H}$, the blue curves are for the textures $\mathrm{P}$, the green curves are for the textures $\mathrm{L}$ and the black curves are for the textures $\mathrm{R}$.

We can observe in figure 3 , that the KSF can discriminate more or less the different structure, the curve KSF has a similar shape for each texture group, but the level is different.

\subsection{Artifacts Detection Using Structure Function Approach}

To detect artifacts using Kolmogorov Structure Function (KSF), the first step is to watch the behavior of the KSF curve for images with artifacts and images without artifacts. One aspect to consider is how to generate the candidates for the necessary space $\mathrm{S}$ as indicated in section 2.3. For this purpose, we have generated the candidates using 2 methods: Candidates generation by JPEG lossy compression and Candidates generation by genetic algorithm as in [5].

In figure 4, we show the results KSF curve for different patches of a satellite image, city environmental with aliasing in some parts, in figure 4(a) we use jpeg lossy compression and in figure 4(b) we use genetic algorithm. The red curves represent the patches with artifacts and the blue curves represent the patches without artifacts.

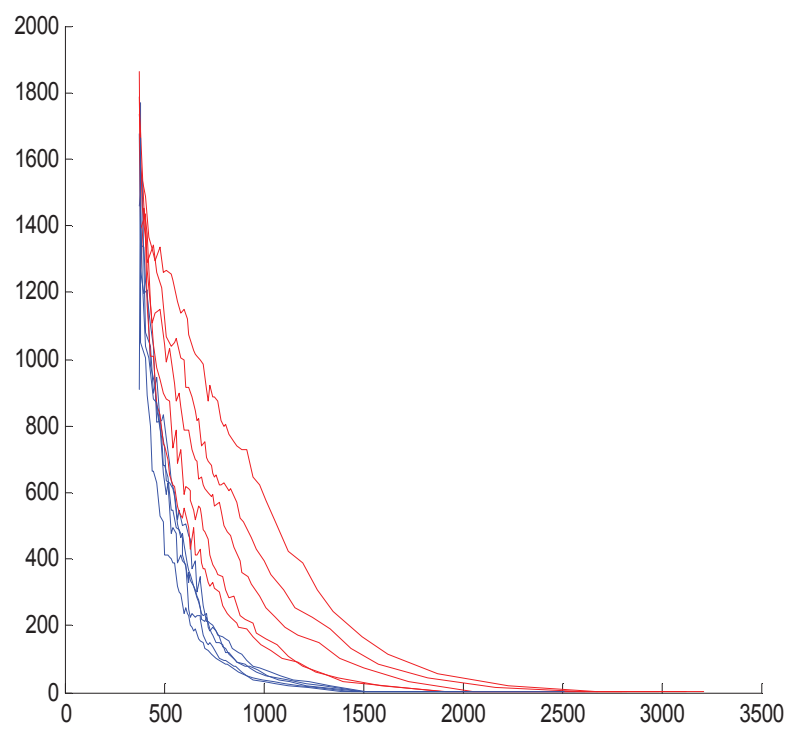

(a) KSF using jpeg lossy compression

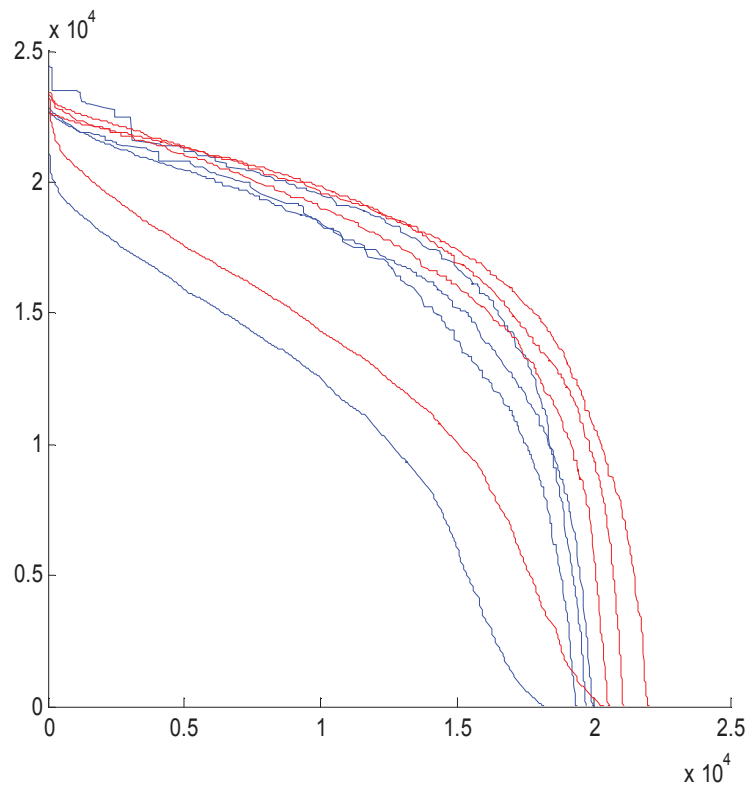

(b) KSF using genetic algorithm

Fig. 4 Kolmogorov Structure Function: x-axis is compressed size of the image (approximation to Kolmogorov Complexity) and yaxis is a structure function. The red curves are for patches with artifacts and the blue curves are for the patches without artifacts.

We can observe that the better discrimination is done when we generate the candidates for the space $S$ using the JPEG lossy compression. Also using JPEG lossy compression the approximation to the Rate-distortion analysis is better.

Having this consideration, we use the jpeg lossy compression for generate candidates and to draw the Kolmogorov Structure Function for each patch of a satellite image and try to detect the artifacts. For this experiments we 
use an image with aliasing introduces manually, in figure 5 we can see the result for aliasing detection.
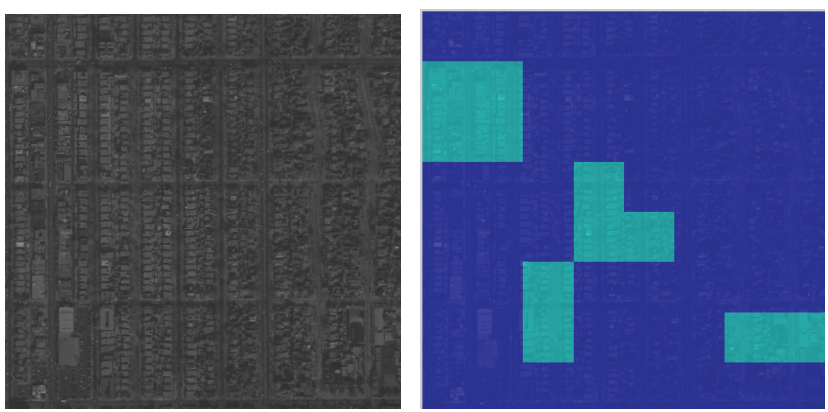

Fig. 5 Aliasing detection in city environmental using KSF and candidate generation with JPEG lossy compression.

The artifacts detection presented in figure 5 was done correctly, the satellite image is an image in city environmental with synthetic artifact as aliasing introduced manually following the follow process: For simulating aliasing in an image, is necessary a downsampling process of the image, while we do the downsampling, aliasing occurs and to avoid aliasing, is necessary apply a low pass filter before downsampling process, finally we combine some part of the image with aliasing and some part of the image without aliasing.

\section{CONCLUSIONS}

The Kolmogorov structure function represents the relationship between an element or data with its model, structure, or explanation.

In this work, we have used the Kolmogorov structure function as a approximation of rate-distortion function using Kolmogorov complexity theory and the complexity-distortion theory, so we can examine the complexity of the images to be analyzed, this complexity would be related to the presence or absence of artifacts.

The generation of candidates for to calculation the Kolmogorov structure function is an important step, in this work was done experiments using 2 methods, generation of candidates by jpeg lossy compression and candidate generation using genetic algorithms, we obtain better results using lossy jpeg compression.

\section{ACKNOWLEDGMENT}

The authors would like to thank Dr. Steven Rooij for provide us the genetic algorithm for to compute the Kolmogorov Structure Function.

\section{REFERENCES}

[1] A. Roman-Gonzalez, M. Datcu; "Parameter Free Image Artifacts Detection: A Compression Based Approach", 2010 SPIE Remote Sensing, vol. 7830, 783008, Toulouse France, September 2010.

[2] A. Mallet, M. Datcu, "Rate Distortion Based Detection of Artifacts in Earth Observation Images", IEEE Geosciences and Remote Sensing Letters, vol. 5, ํㅜ 3, pp. 354-358, July 2008.

[3] N. K. Vereshchagin, P. M. B. Vitanyi; "Kolmogorov's Structure Functions and Model Selection"; IEEE Transaction on Information Theory, Vol. 50, $\mathrm{N}^{\circ} 12$, December 2004, pp. 3265-3290.

[4] N. K. Vereshchagin, P. M. B. Vitanyi; "Rate Distortion and Denoising of Individual Data Using Kolmogorov Complexity"; IEEE Transaction on Information Theory, Vol. $56, \mathrm{~N}^{\circ} 7$, July 2010, pp. 3438-3454.

[5] S. de Rooj, P. M. B. Vitanyi; "Approximating RateDistortion Graphs of Individual Data: Experiments in Lossy Compression and Denoising"; CWI.

[6] D. M. Sow, A. Eleftheriadis; "Complexity Distortion Theory"; IEEE Transaction on Information Theory, Vol. 49, $\mathrm{N}^{\circ} 3$, March 2003, pp. 604-608.

[7] N. Vereshchagin, P. Vitanyi; "Kolmogorov's Structure Functions with an Application to the Foundations of Model Selection"; Proceedings of the $43^{\text {rd }}$ Annual IEEE Symposium on Foundations of Computer Science, 2002, pp. 1-10.

[8] The USC-SIPI Image Database, [online] available at http://spici.usc.edu/database. 